\title{
Cyclicity of Special Operators on a BK with AK Space
}

\author{
Leila Bagheri and Bahmann Yousefi \\ Department of Mathematics, Payame Noor University, P.O. Box 19395-3697, Tehran, Iran
}

Correspondence should be addressed to Bahmann Yousefi; b_yousefi@pnu.ac.ir

Received 12 July 2015; Accepted 1 December 2015

Academic Editor: Harumi Hattori

Copyright (C) 2015 L. Bagheri and B. Yousefi. This is an open access article distributed under the Creative Commons Attribution License, which permits unrestricted use, distribution, and reproduction in any medium, provided the original work is properly cited.

Let $\Omega$ be a complex domain and let $F$ be a reflexive BK space with AK such that $\widehat{F} \subset H(\Omega)$ and the functional of evaluation at $\lambda$ is bounded for all $\lambda \in \Omega$. We will investigate the cyclicity for the adjoint of a weighted composition operator acting on $\widehat{F}$.

\section{Introduction}

We write $\omega$ for the set of all complex sequences $x=\left(x_{k}\right)_{k=0}^{\infty}$ Let $\phi$ denote the set of all finite sequences. By $e^{(n)}$, we denote the sequence with $e_{n}^{(n)}=1$ and $e_{k}^{(n)}=0$ whenever $k \neq n$. For any sequence $x=\left(x_{k}\right)_{k=0}^{\infty}$, let $x^{[n]}=\sum_{k=0}^{n} x_{k} e^{(k)}$ be its $n$-section. Given any subset $F$ of $\omega$, we write $\widehat{F}$ for the set of all formal power series $\widehat{f}$ with $\widehat{f}(z)=\sum_{k=0}^{\infty} f_{k} z^{k}$ where $f=$ $\left(f_{k}\right)_{k=0}^{\infty} \in F$, regardless of whether or not the series converges for any value of $z$. If $\widehat{F}$ endowed with the norm of $F$, then $F$ and $\widehat{F}$ are norm isomorphic. Let $\widehat{M}_{z}: \widehat{F} \rightarrow \widehat{\omega}$ be defined by $\left(\widehat{M}_{z} \widehat{f}\right)=\sum_{k=0}^{\infty} f_{k} z^{k+1}$, so the corresponding shift operator $M: F \rightarrow \omega$ is defined by $(M f)_{n}=f_{n-1}$ if $n \geq 1$ and 0 else.

A BK space is a Banach sequence space with the property that convergence implies coordinatewise convergence. A BK space $F$ containing $\phi$ is said to have AK if every sequence $f=$ $\left(f_{k}\right)_{k=0}^{\infty} \in F$ has a unique representation $f=\sum_{k=0}^{\infty} f_{k} e^{(k)}$; that is, $f=\lim _{n \rightarrow \infty} f^{[n]}$; it is said to have $\mathrm{AD}$, if $\phi$ is dense in $F$. Given any subset $F$ of $\omega$, the set

$$
F^{\beta}=\left\{a \in \omega: \sum_{k=0}^{\infty} a_{k} f_{k} \text { converges for all } f \in F\right\}
$$

is called the $\beta$-dual of $F$.

If $\lambda$ is a complex number, then $e(\lambda)$ denotes the functional of evaluation at $\lambda$, defined on the polynomials $p$ by $e(\lambda)(\widehat{p})=$ $\widehat{p}(\lambda)$. A point $\lambda$ is said to be a bounded point evaluation on $\widehat{F}$ if the functional $e(\lambda)$ extends to a continuous linear functional on $\widehat{F}$. Finally, we consider the multiplication of formal power series $\widehat{h}=\widehat{f} \widehat{g}$ given by

$$
\widehat{h}(z)=\sum_{k=0}^{\infty} h_{k} z^{k}=\left(\sum_{k=0}^{\infty} f_{k} z^{k}\right)\left(\sum_{k=0}^{\infty} g_{k} z^{k}\right),
$$

where $h_{k}=\sum_{j=0}^{k} f_{j} g_{k-j}$ for all integers $k \geq 0$. If $\widehat{f} \in \widehat{F}$ and $\widehat{p}$ is a polynomial, then to the vector $\widehat{p}\left(M_{z}\right) \widehat{f}$ the formal power series $\widehat{p}(z) \hat{f}(z)$ corresponds.

Let $\Omega$ be a complex domain and let $\widehat{F}$ be a Banach space of formal power series with coefficients in a reflexive BK space with AK such that $\widehat{F} \subset H(\Omega)$. It is convenient and helpful to introduce the notation $\left\langle x, x^{*}\right\rangle$ to stand for $x^{*}(x)$, for $x \in \widehat{F}$ and $x^{*} \in(\widehat{F})^{*}$. We assume $1 \in \widehat{F}$ and the operators $\widehat{M}_{z}$ and the functional $e(\lambda)$ of evaluation at $\lambda(\lambda \in \Omega)$ are bounded on $\widehat{F}$.

A complex valued function $\varphi$ on $\Omega$ for which $\varphi \widehat{f} \in \widehat{F}$ for every $\widehat{f} \in \widehat{F}$ is called a multiplier of $\widehat{F}$ and the collection of all of these multipliers is denoted by $\mathscr{M}(\widehat{F})$. If $\widehat{M}_{z}$ is a bounded operator on $\widehat{F}$, the adjoint $\left(\widehat{M}_{z}\right)^{*}:(\widehat{F})^{*} \rightarrow(\widehat{F})^{*}$ satisfies $\left(\widehat{M}_{z}\right)^{*} e(\lambda)=\lambda e(\lambda)$. In general each multiplier $\varphi$ of $\widehat{F}$ determines a multiplication operator $\widehat{M}_{\varphi}$ defined by $\widehat{M}_{\varphi} \widehat{f}=$ $\varphi \widehat{f}, \widehat{f} \in \widehat{F}$. Also $\left(\widehat{M}_{\varphi}\right)^{*} e(\lambda)=\varphi(\lambda) e(\lambda)$. It is well-known that each multiplier is a bounded analytic function on $\Omega$. Indeed $|\varphi(\lambda)| \leq\left\|\widehat{M}_{\varphi}\right\|$ for each $\lambda$ in $\Omega$.

Let $\varphi$ be an analytic self-map of the open unit disk $U$. A composition operator $C_{\varphi}$ maps an analytic function $\widehat{f} \in \widehat{F}$ into $\left(C_{\varphi} \widehat{f}\right)(z)=\widehat{f}(\varphi(z))$. If $w \in \mathscr{M}(\widehat{F})$ and $C_{\varphi}$ is bounded, 
$M_{w} C_{\varphi}$ is called a weighted composition operator on $\mathscr{M}(\widehat{F})$. By $\varphi_{n}$ we will mean the $n$th iterate of $\varphi$.

Let $X$ be a Banach space. We denote by $B(X)$ the set of bounded operators on the Banach space $X$. Let $A \in B(X)$ and $x \in X$. We say that $x$ is a cyclic vector of $A$ if $X$ is equal to the closed linear span of the set $\left\{A^{n} x: n=0,1,2, \ldots\right\}$. An operator $A \in B(X)$ is called cyclic if it has a cyclic vector. In this paper, we investigate the cyclicity of weighted composition operators on some BK spaces with AK.

\section{Main Results}

The sequence spaces has been the focus of attention for several decades and many properties of operators on these spaces have been studied (e.g., [1]). For $p=\left(p_{k}\right)_{k}^{\infty}$, a sequence with $p_{k}>0$ for all $k$, Malkowsky considered the space $b v(p)=\left\{x \in \omega: \sum_{k=0}^{\infty}\left|x_{k}-x_{k-1}\right|^{p_{k}}<\infty\right\}$ and studied its $\beta$-dual and characterized some linear operators on $b v(p)$ [2]. In [3] the reflexivity of $\Lambda$-summable sequences from a Banach space is investigated whenever $\Lambda$ is a Banach perfect sequence space. Some BK spaces including the spaces $\mathcal{c}_{0}$ and $c$ have been introduced in [4] and also their duals have been computed. In [5], Aydin and Basar have introduced new classes of sequence spaces which include the spaces $l_{p}$ and $l_{\infty}$, and the characterization of some other classes of sequence spaces have also been derived. Malafosse has given some properties and applications of Banach algebras of bounded operators $B(X)$, when $X$ is a BK space [6]. In [7], Mursaleen and Noman introduced some spaces of difference sequences which are the BK spaces of nonabsolute type and proved that these spaces are linearly isomorphic to the spaces $c_{0}$ and $c$, respectively. In $[8,9]$, Mursaleen and Noman established some identities or estimates for the operator norms and the Hausdorff measures of noncompactness of certain operators on some BK spaces of weighted means have been investigated. Furthermore, by using the Hausdorff measure of noncompactness, they applied their results to characterize some classes of compact operators on those spaces. In [10] some identities or estimates for the operator norms and the Hausdorff measure of noncompactnesss of certain matrix operators on some BK spaces have been established. In [11], Basarir and Kara have characterized some classes of compact operators on special normed Riesz sequence spaces by using the Hausdorff measure of noncompactness. A characterization of compact operators between certain BK spaces has been given by Malkowsky in [12]. Also, Malkowsky gave general bounded linear operators on special BK spaces that are strongly summable to 0 , summable and bounded with index equal to or greater than 1 [13]. In [14], Kirisci gave well-known result related to some properties, dual spaces, and matrix transformations of the sequence space $b v$ and introduced the matrix domain of space $b v$ with arbitrary triangle matrix. The reflexivity of multiplication operators on some BK spaces with AK property has been studied in [15]. Cyclicity of the adjoint of weighted composition operators on Hilbert function spaces, Fock spaces, and weighted Hardy spaces has been studied in [16-18]. In this section we want to study the cyclicity of the adjoint of a weighted composition operator acting on a space of formal power series with coefficients in a BK space with AK property. By the notations $\sigma_{p}\left(\widehat{M}_{z}^{*}\right)$ and $U$ we mean the point spectrum of $\widehat{M}_{z}^{*}$ and the open unit disc, respectively.

Theorem 1. Let $F$ be a BK space with $A K$. A complex number $\lambda$ is a bounded point evaluation on $\widehat{F}$ if and only if $\lambda \in \sigma_{p}\left(\widehat{M}_{z}^{*}\right)$ and if and only if $\left\{\lambda^{n}\right\}_{n=0}^{\infty} \in F^{\beta}$.

Proof. If $\lambda$ is a bounded point evaluation it is clear that $\lambda \epsilon$ $\sigma_{p}\left(\left(\widehat{M}_{z}\right)^{*}\right)$. Conversely, let $\lambda \in \sigma_{p}\left(\widehat{M}_{z}^{*}\right)$ and $g^{\lambda}=\left\{g_{n}^{\lambda}\right\}_{n=0}^{\infty} \in$ $F^{\beta}$ be a corresponding eigenvector in $(\widehat{F})^{*}$. For $f \in F$, we have

$$
\begin{aligned}
\left\langle\widehat{M}_{z} \widehat{f},\left(g^{\lambda}\right)^{\wedge}\right\rangle & =\left\langle\widehat{f},\left(\widehat{M}_{z}\right)^{*}\left(g^{\lambda}\right)^{\wedge}\right\rangle=\left\langle\widehat{f}, \lambda\left(g^{\lambda}\right)^{\wedge}\right\rangle \\
& =\lambda\left\langle\widehat{f},\left(g^{\lambda}\right)^{\wedge}\right\rangle .
\end{aligned}
$$

Hence for all $n$, we get

$$
\begin{aligned}
\left\langle\left(e^{(n+1)}\right)^{\wedge},\left(g^{\lambda}\right)^{\wedge}\right\rangle & =\left\langle\widehat{M}_{z}\left(e^{(n)}\right)^{\wedge},\left(g^{\lambda}\right)^{\wedge}\right\rangle \\
& =\lambda^{n+1}\left\langle\left(e^{(0)}\right)^{\wedge},\left(g^{\lambda}\right)^{\wedge}\right\rangle
\end{aligned}
$$

and so $g_{n+1}^{\lambda}=\lambda^{n+1} g_{0}^{\lambda}$. Since $g^{\lambda} \neq 0$ we have $g_{0}^{\lambda} \neq 0$. Put $E(\lambda)=\left(g^{\lambda}\right)^{\wedge} / g_{0}^{\lambda}$. Then for all $n$ we have $\left\langle\left(e^{(n+1)}\right)^{\wedge}, E(\lambda)\right\rangle=$ $\lambda^{n+1}$ and this implies that $\langle\widehat{p}, E(\lambda)\rangle=\widehat{p}(\lambda)$ for all polynomials p. Since polynomials are dense in $\widehat{F}, \lambda$ is a bounded point evaluation and intact $E(\lambda)=e(\lambda)$. Now it is clear that $\lambda$ is a bounded point evaluation if and only if $\left\{\lambda^{n}\right\}_{n=0}^{\infty} \in F^{\beta}$.

Theorem 2. Let $F$ be a BK space with $A K$ and $A D$ such that each point of $U$ is a bounded point evaluation on $\widehat{F}$. Then a polynomial $\widehat{p}$ is cyclic for $\widehat{M}_{z}$ if and only if $\widehat{p}$ vanishes at no point in $U$.

Proof. Let $\widehat{p}(z)=\left(z-\lambda_{1}\right) \cdots\left(z-\lambda_{m}\right)$ be such that $\lambda_{i} \notin U$ for $i=1, \ldots, m$. Fix $k \in\{1, \ldots, m\}$ and consider $M_{k} \in(\widehat{F})^{*}$ satisfying $M_{k}\left(\widehat{M}_{z}\right)^{n}\left(z-\lambda_{k}\right)=0$ for all integers $n \geq 0$. Since $F^{*}=F^{\beta}$, there exists $h^{(k)} \in F^{\beta}$ such that $M_{k} \widehat{f}=\langle\widehat{f}, \widehat{h}(k)\rangle$ for all $f \in F$. Note that

$$
\begin{aligned}
M_{k}\left(\widehat{M}_{z}\right)^{n}\left(z-\lambda_{k}\right) & =M_{k}\left(z^{n+1}-\lambda_{k} z^{n}\right) \\
& =h_{n+1}^{(k)}-\lambda_{k} h_{n}^{(k)}
\end{aligned}
$$

for all integers $n \geq 0$. Since $M_{k}\left(\widehat{M}_{z}\right)^{n}\left(z-\lambda_{k}\right)=0$, we get $h_{n+1}^{(k)}=\lambda_{k} h_{n}^{(k)}$ and so $h_{n+1}^{(k)}=\lambda_{k}^{n+1} h_{0}^{(k)}$ for all $n \geq 0$. But $\left\{\lambda_{k}^{n}\right\}_{n} \notin$ $F^{\beta}$ and $h^{(k)} \in F^{\beta}$; hence $h_{n}^{(k)}=0$ for all $n$ and so $M_{k}=0$. Thus by the Hahn Banach Theorem, $z-\lambda_{k}$ is cyclic for $k=$ $1, \ldots, m$ and so $\widehat{p}(z)$ is a cyclic vector for $\widehat{M}_{z}$. The converse case is clear.

Theorem 3. Suppose that $F$ is a $B K$ space with $A K$ and $A D$, $M(\widehat{F})=H^{\infty}, \varphi$ is an analytic function on $U$ satisfying $\|\varphi\|_{U}<$ 1 , and $\widehat{w} \in \mathscr{M}(\widehat{F})$. Also, let $\{e(z): z \in U\}$ be bounded and there exists $z_{0} \in U$ satisfying $\widehat{w}\left(\varphi_{k}\left(z_{0}\right)\right) \neq 0$ for all $k \geq 0$, and 
assume that the set $\left\{\varphi_{k}\left(z_{0}\right): k \geq 0\right\}$ has a limit point in $U$. Then $e\left(z_{0}\right)$ is a cyclic vector for the operator $\left(\widehat{M}_{\widehat{w}} C_{\varphi}\right)^{*}$ acting on $\widehat{F}^{\beta}$.

Proof. Let the map $L: \mathscr{M}(\widehat{F}) \rightarrow B(\widehat{F})$ be given by $L(\widehat{\psi})=$ $\widehat{M}_{\widehat{\psi}}$. We prove that $L$ is continuous. For this we use the closed graph theorem. Suppose $\widehat{\psi}_{n}$ converges to $\widehat{\psi}$ in $\mathscr{M}(\widehat{F})$ and $L\left(\widehat{\psi}_{n}\right)=\widehat{M}_{\widehat{\psi}_{n}}$ converges to $A$ in $B(\widehat{F})$. Then, for each $f$ in F,

$$
A \widehat{f}=\lim _{n} \widehat{M}_{\widehat{\psi}_{n}} \widehat{f}=\lim _{n} \widehat{\psi}_{n} \widehat{f} .
$$

Thus $\left\{\widehat{\psi}_{n} \widehat{f}_{n}\right.$ is convergent in $\widehat{F}$. Now by the continuity of point evaluations $\widehat{\psi}_{n} \widehat{f}$ converges pointwise to $\widehat{\psi} \widehat{f}$ on $U$. So $A \widehat{f}$ is analytic and agrees with $\widehat{\psi} \widehat{f}$ on $U$. Hence $A \widehat{f}=\widehat{\psi} \widehat{f}$ and $A=\widehat{M}_{\widehat{\psi}}$. Therefore, $L$ is continuouse and there is a constant $c$ such that $\left\|\widehat{M}_{\widehat{\psi}}\right\| \leq c\|\widehat{\psi}\|_{U}$ for all $\widehat{\psi}$ in $\mathscr{M}(\widehat{F})$. But $\|\widehat{\psi}\| \leq\left\|\widehat{M}_{\widehat{\psi}}\right\|$ for all $\widehat{\psi}$ in $\mathscr{M}(\widehat{F})$. Thus $\|\widehat{\psi}\| \leq c\|\widehat{\psi}\|_{U}$ for all $\psi \in \mathscr{M}(\widehat{F})$. Since $\varphi \in H^{\infty}$ and $\mathscr{M}(\widehat{F})=H^{\infty}$ we will use $\widehat{\varphi}$ instead of $\varphi$. Let $f \in F$; then $C_{\widehat{\phi}} \widehat{f}=\widehat{f} \circ \widehat{\varphi} \in H^{\infty}$ since $\|\widehat{\varphi}\|_{U}<1$. So

$$
\|\widehat{f} \circ \widehat{\varphi}\| \leq c\|\widehat{f} \circ \widehat{\varphi}\|_{U} \leq c\|\widehat{f}\|_{U},
$$

since $\widehat{\varphi}(U) \subseteq U$. On the other hand, note that, for all $f$ in $F,\|\widehat{f}\|_{U} \leq \gamma\|\widehat{f}\|$, where $\gamma=\sup \{\|e(z)\|: z \in U\}$. Now we get $\left\|C_{\hat{\varphi}} \hat{f}\right\| \leq c \gamma\|\hat{f}\|$, which implies that $C_{\widehat{\varphi}}$ and so $\widehat{M}_{\widehat{w}} C_{\widehat{\varphi}}$ are bounded. Now, put $A=\widehat{M}_{\widehat{w}} C_{\widehat{\varphi}}$. To complete the proof we show that if $\left\langle\widehat{g},\left(A^{*}\right)^{k} e\left(z_{0}\right)\right\rangle=0$ for all $k \geq 0$ then $\hat{g}$ should be the zero constant function. For this note that

$$
\left\langle\widehat{g},\left(A^{*}\right)^{k} e\left(z_{0}\right)\right\rangle=\left(\prod_{i=0}^{k-1} \overline{\widehat{w}\left(\widehat{\varphi}_{i}\left(z_{0}\right)\right)}\right) \widehat{g}\left(\widehat{\varphi}_{k}\left(z_{0}\right)\right) .
$$

By the assumptions, clearly we get $\widehat{g}\left(\widehat{\varphi}_{k}\left(z_{0}\right)\right)=0$ for all $k \geq 0$. Since $\left\{\widehat{\varphi}_{k}\left(z_{0}\right): k \geq 0\right\}$ has limit point in $U$, it should be $\widehat{g}=0$. Thus, $e\left(z_{0}\right)$ is a cyclic vector for the operator $\left(\widehat{M}_{\widehat{w}} C_{\widehat{\varphi}}\right)^{*}$ acting on $\widehat{F}^{\beta}$. This completes the proof.

\section{Conflict of Interests}

The authors declare that there is no conflict of interests regarding the publication of this paper.

\section{References}

[1] A. Wilansky, Summability through Functional Analysis, Mathematics Studies 85, North-Holland, 1984.

[2] E. Malkowsky, "Linear operators in certain BK spaces," Bolyai Society Mathematical Studies, vol. 5, pp. 259-273, 1996.

[3] M. A. Ould Sidaty, "Reflexivity and AK-property of certain vector sequence spaces," Bulletin of the Belgian Mathematical Society, vol. 10, no. 4, pp. 579-583, 2003.

[4] C. Aydin and F. Başar, "On the new sequence spaces which include the spaces $c_{0}$ and $c$," Hokkaido Mathematical Journal, vol. 33, no. 2, pp. 383-398, 2004.

[5] C. Aydin and F. Basar, "Some new sequence spaces which include the spaces $\ell_{p}$ and $\ell_{\infty}$," Demonstratio Mathematica, vol. 38 , no. 3, pp. 641-656, 2005.
[6] B. D. Malafosse, "The Banach algebra $B(X)$ and Applications, where $X$ is a BK space," Matematički Vesnik, vol. 57, pp. 41-60, 2005.

[7] M. Mursaleen and A. K. Noman, "On some new difference sequence spaces of non-absolute type," Mathematical and Computer Modelling, vol. 52, no. 3-4, pp. 603-617, 2010.

[8] M. Mursaleen and A. K. Noman, "Applications of the Hausdorff measure of noncompactness in some sequence spaces of weighted means," Computers \& Mathematics with Applications, vol. 60, no. 5, pp. 1245-1258, 2010.

[9] M. Mursaleen and A. K. Noman, "Compactness by the Hausdorff measure of noncompactness," Nonlinear Analysis, vol. 73, no. 8, pp. 2541-2557, 2010.

[10] M. Mursaleen and A. K. Noman, "The Hausdorff measure of noncompactness of matrix operators on some BK spaces," Operators and Matrices, vol. 5, no. 3, pp. 473-486, 2011.

[11] M. Basarir and E. E. Kara, "On compact operators on the Riesz $B^{m}$-difference sequences," Iranian Journal of Science and Technology, vol. 3, pp. 371-376, 2012.

[12] E. Malkowsky, "Characterization of compact operators between certain BK spaces," Filomat, vol. 27, no. 3, pp. 447-457, 2013.

[13] E. Malkowsky, "Measure of noncompactness for compact matrix operators on some BK spaces," Filomat, vol. 28, no. 5, pp. 1081-1086, 2014.

[14] M. Kirisci, "The sequence space bv and some applications," Mathematica Aeterna, vol. 4, no. 3, pp. 207-223, 2014.

[15] L. Bagheri and B. Yousefi, "Reflexivity of the shift operator on some BK spaces," Rendiconti del Circolo Matematico di Palermo, vol. 63, no. 1, pp. 91-96, 2014.

[16] Z. Kamali, B. Khani Robati, and K. Hedayatian, "Cyclicity of the adjoint of weighted composition operators on the Hilbert space of analytic functions," Czechoslovak Mathematical Journal, vol. 61, no. 2, pp. 551-563, 2011.

[17] W. U. Shu-Hong, "Cyclic behavior for adjoint operators of composition operators on the weighted Hardy space," Guangxi Sciences, vol. 15, no. 4, pp. 139-151, 2008.

[18] L. Zhao, "Unitary weighted composition operators on the Fock space of $c^{n}$," Complex Analysis and Operator Theory, vol. 8, no. 2, pp. 581-590, 2014. 


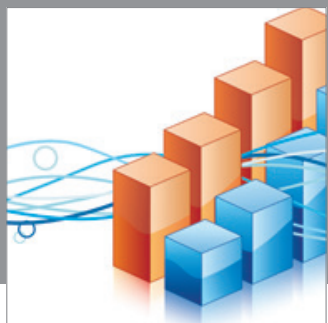

Advances in

Operations Research

mansans

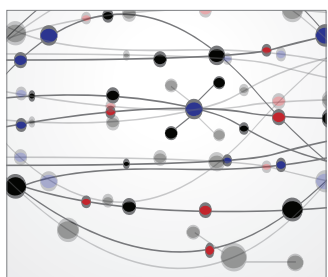

The Scientific World Journal
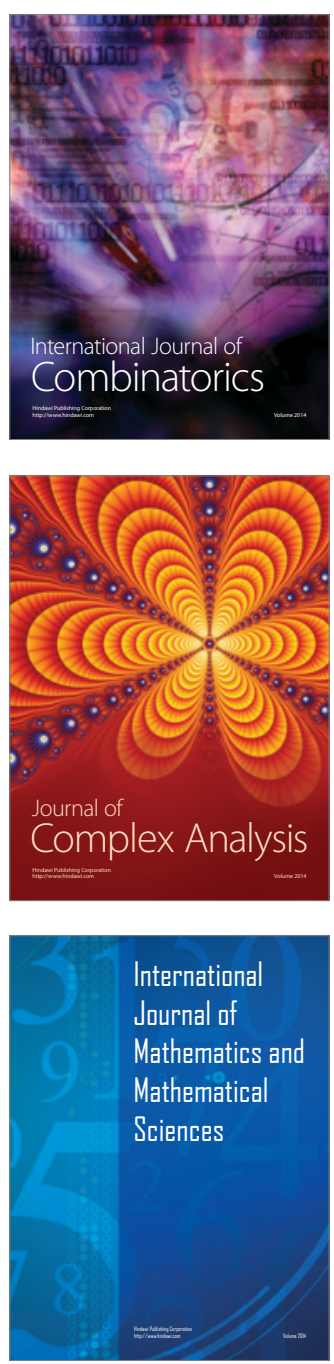
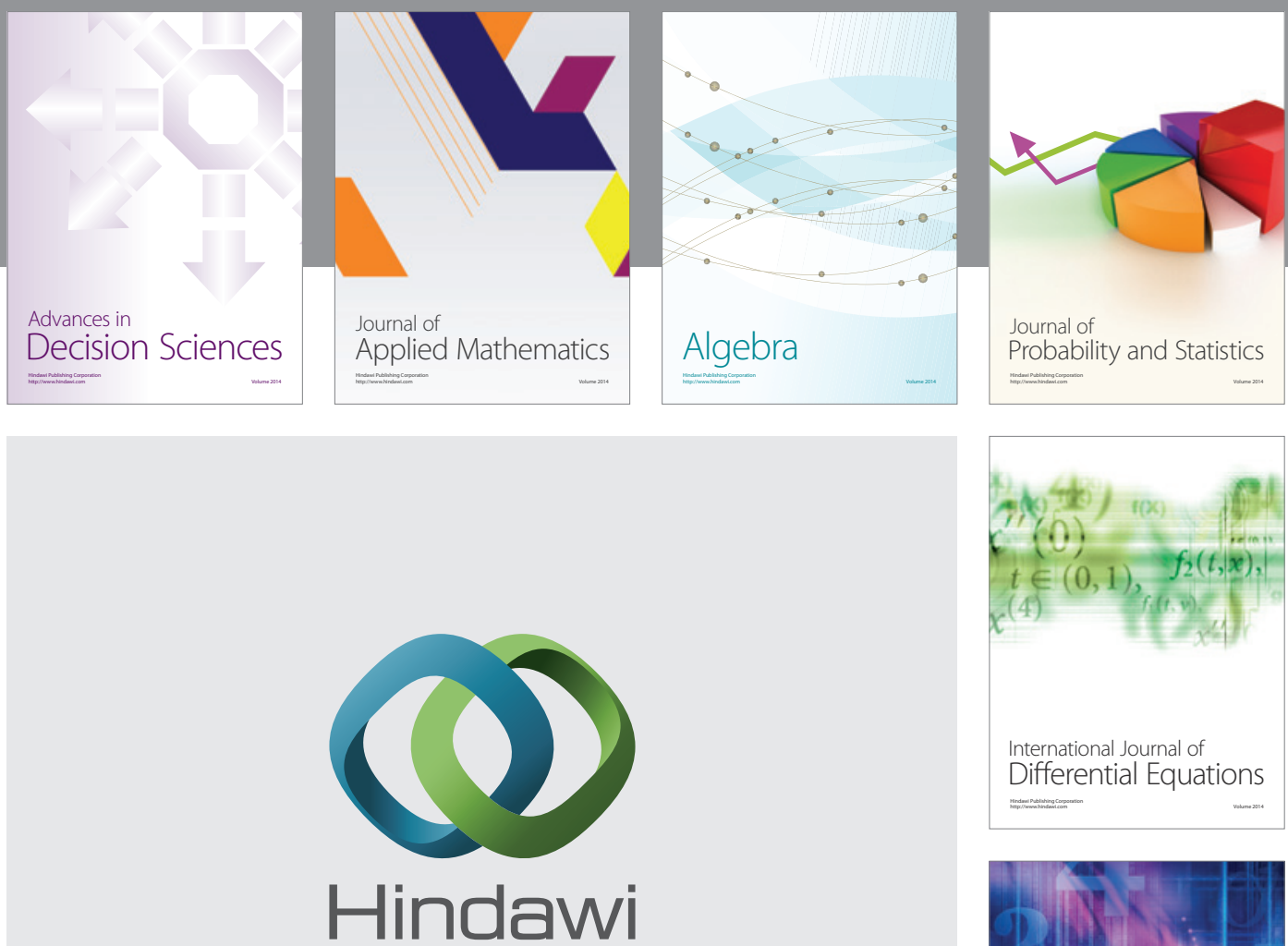

Submit your manuscripts at http://www.hindawi.com
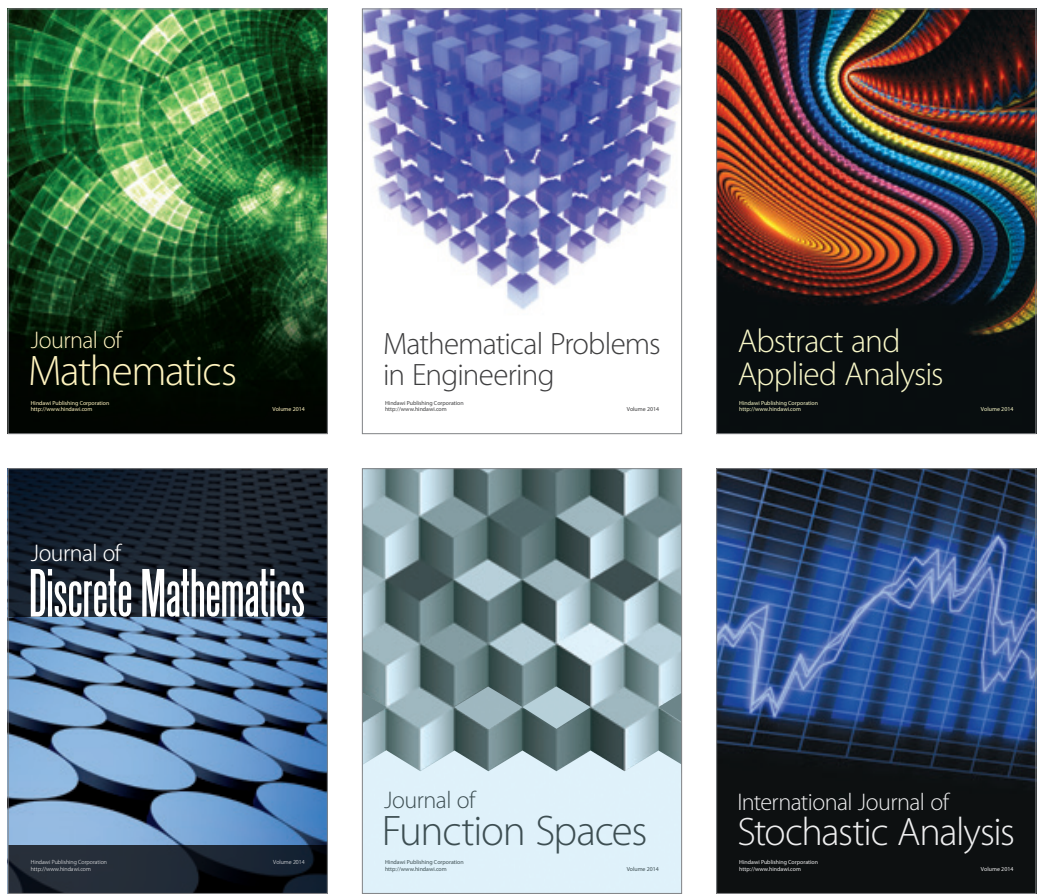

Journal of

Function Spaces

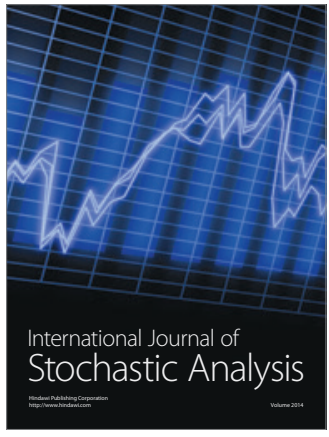

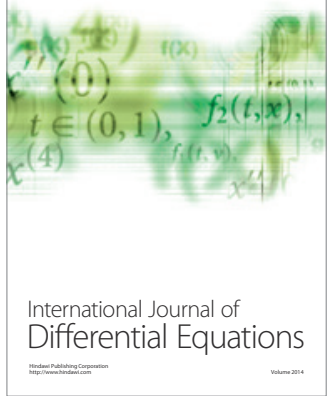
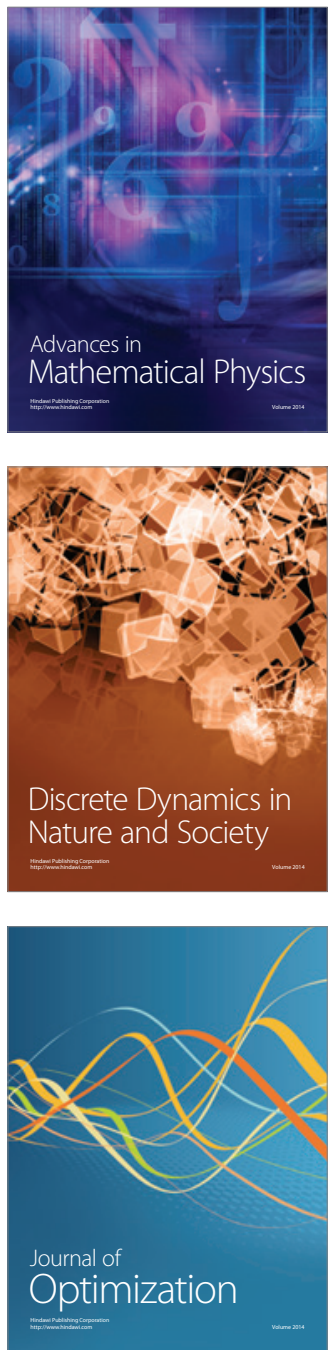